\title{
MORPHOLOGICAL STUDY OF COMMUNICATION BETWEEN THE LONGITUDINAL STRIPS AND THE HUMAN CORPUS CALLOSUM
}

\author{
O. D. Boiagina \\ KHARKIV NATIONAL MEDICAL UNIVERSITY, KHARKIV, UKRAINE
}

Background. It is established that there is an anatomical relationship between the corpus callosum and longitudinal strips. These formations must correlate to the common commissural system of the corpus callosum conductors. At present this issue in such a formulation is not considered in literature on Neuromorphology.

Objective. The study was aimed to determine the commutations between the longitudinal strips and commissural conductors of the corpus callosum.

Methods. The corpus callosum of people aged from 36 to 60 was studied. Some slices of the corpus callosum stem were used for impregnation and then inserted in paraffin blocks. Another part of these slices was subjected to plastination in epoxy resin.

Results. Lateral longitudinal strips contain significantly greater mass of nerve conductors. Most of them compactly pass along limbic ring, while the other part is combined to nerve fibres of the corpus callosum commissural cords. The longitudinal strips are coated with an outer limiting glial membrane (grey coating).

Conclusions. By means of the corpus callosum the connections between conscious and subconscious brain areas are structurally fixed. It can be assumed that longitudinal strips have relation to hippocampal area, related to the ancient formation of the pallium. This does not exclude the fact that the nerve fibres, found within longitudinal strips may have projections on the cortical cells of vaulted gyrus, which is considered to be paleopallium sphere. So, this interaction between the ancient and the old cortex should presumably be carried out by means of lateral longitudinal strips.

KEY WORDS: the corpus callosum; lateral longitudinal stripes; medial longitudinal stripes.

\section{Introduction}

It is established that there is a direct anatomical connection between the corpus callosum and such formations as longitudinal strips (medial and lateral), fornix and transparent partition [1, 4-7]. According to current researches, they are considered to be conductive formations of limbic brain. It is established that the longitudinal strips are thin strands of nerve fibres encircling upper surface of the corpus callosum, which anteriorly are connected to subcallosal gyrus, and posteriorly under the splenium reach the hippocampal area of dentate gyrus. This fact significantly defines the closed circular structure of limbic brain.

Due to this fact, it is clear that these formations in some way should be linked to a com-

Corresponding author: Olha Boiagina, Department of Human Anatomy, Kharkiv National Medical University, 4 Prospect Nauky, Kharkiv Ukraine, 61022

Phone number: +380675729290

E-mail: olya-boyagina@yandex.ru mon system of commissural conductors of the corpus callosum itself. Despite this evidence, in the current literature on Neuromorphology this issue, in this formulation, has not been even considered. This fact can be surprising, because of its importance in understanding the mediating links in the associative interaction between conscious and subconscious psychic spheres.

We actually started studying these structures in this aspect in our previous research [2] and brought to notice the shape of upper surface of the corpus callosum, having two noteworthy sublitities, known in the literature as the transverse and longitudinal strips. Firstly, we have proved that so called transverse strips are actually an external protrusion of constituent formations of the corpus callosum that we termed as commissural cords. The wavily curved longitudinal strips run on their spindleshaped elevations, and a pair of them in contralateral position occupies borderline position 
between the free part of the corpus callosum and the medial surface of cingulate gyri. In the intermediate position between them there is a pair of closely spaced similar formations, which are called medial longitudinal strips. Besides the detailed description of their form that is provided above in this article, we mention only one significant feature that evidences that these longitudinal strips do not just lie on the upper surface of the corpus callosum but are closely related to it. This is indicated by periodically occurring local immersions of longitudinal strips in the thickness of the corpus callosum in recesses between transverse spindleshaped elevations that gives them the shape of suture stitches. Taking into consideration this persuasive anatomical fact, it is not enough to be absolutely sure in the presence of commutation relations between these formations and commissural conductors of the corpus callosum. To answer this question some additional histological examinations were required.

Our study was aimed to determine the commutation relations between longitudinal strips and commissural conductors of the corpus callosum.

\section{Methods}

In this research we used whole mounts of the corpus callosum of 5 men and 5 women aged from 36 to 60 years, which were separated from whole brain mounts after their twoweek fixation in $10 \%$ neutral formalin. These mounts were received due to the agreement between Kharkiv National Medical University and Kharkiv Regional Bureau of Forensic Medical Examination.

The mounts were used to excise plate slices of standard thickness $(2 \mathrm{~mm})$ out of corpus callosum stem. In addition, their excision was performed in two mutually perpendicular directions: lengthwise and crosswise. Some of them were used for impregnation and further embedding in paraffin blocks, according to the conventional methods. Later they were used to prepare serial slices with traditional hematoxylin and eosin, and Van Gieson staining.

The other part was subjected to plastination in epoxy resin using the epoxy adhesive Khimkontakt-Epoksi, according to the method developed at the Department of Human Anatomy of UMSA (Poltava) [3].

At the end of the last stage of impregnation in pure epoxy resin these plate mounts were placed between two glass plates, isolated with plastic spacers (to prevent adhesion of mounts and glass). In such a 'sandwich' mounts were thrust together with sparing clips and in this way they were subjected to final polymerization and acquired flattened, but not deformed shape.

After complete polymerization these palatinate mounts were used for making thin sections that were stained with a $1 \%$ solution of methylene blue per $1 \%$ solution of borax.

The study of all mounts (paraffin slices and thin epoxy sections) was carried out using a binocular microscope MBS-9 and Konus microscope with a digital photo-adaptor.

\section{Results}

When staining thin epoxy sections of the corpus callosum with methylene blue it was found out that lateral longitudinal strips, which shape is of pointed hills in cross-section, have basophilia of the same intensity as that of its main stratum. However, there is a layer of a light coloured matter between them, giving the reason to assume certain detachment between them (Fig. 1) and we have to find out the extent of this detachment.

Medial longitudinal strips located in the medial position of corpus callosum cross section look somewhat different (Fig. 2). Because of their irregular location on the surface of spindle-shaped elevations, that was noted in the introductory part of this article, in crosssectional perspective they are not always detected as paired or equal in terms of their altitude formations. Therefore, the density of their connection with the substance of the corpus callosum is a kind of an ambiguous judgment. For example, in some of its epoxy slices (in cross section) they look like elevations of asymmetrical height and their substance (in methylene

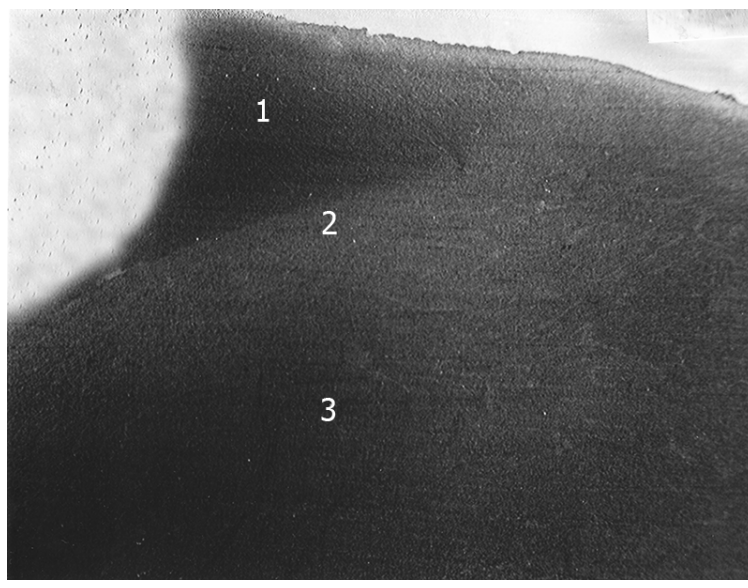

Fig. 1. Lateral cross-section of an adult male corpus callosum. Epoxy slice. Painted in methylene blue. $x 7$. 1 - lateral longitudinal strip in cross-section; 2 - intermediate area; 3 - the substance of the corpus callosum. 


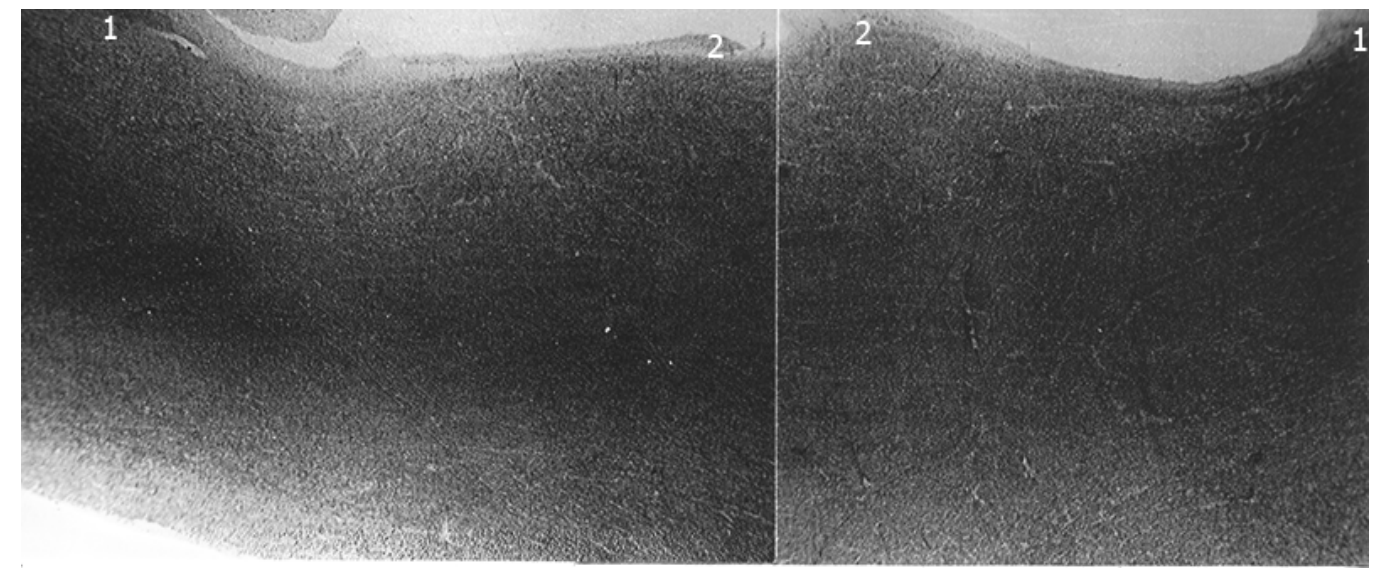

Fig. 2. The cross-section of the corpus callosum of a 56-year-old male. Epoxy slice. Painted in methylene blue. x4. 1 - lateral longitudinal strips; 2 - medial longitudinal strips.

blue staining) has less pronounced basophilia compared to the basic thickness of the corpus callosum (Fig. 2). In addition, there is a poorly pronounced intermittent light streak between the corpus callosum and medial strips.

These tinctorial differences between the substance of medial longitudinal strips and the actual corpus callosum can be distinctly detected on paraffin slices when stained by Van Gieson (Fig. 3). First of all it should be noted that both substances are inseparably adjusted to each other, but between them there is a visible intermediate area in the form of a nar-

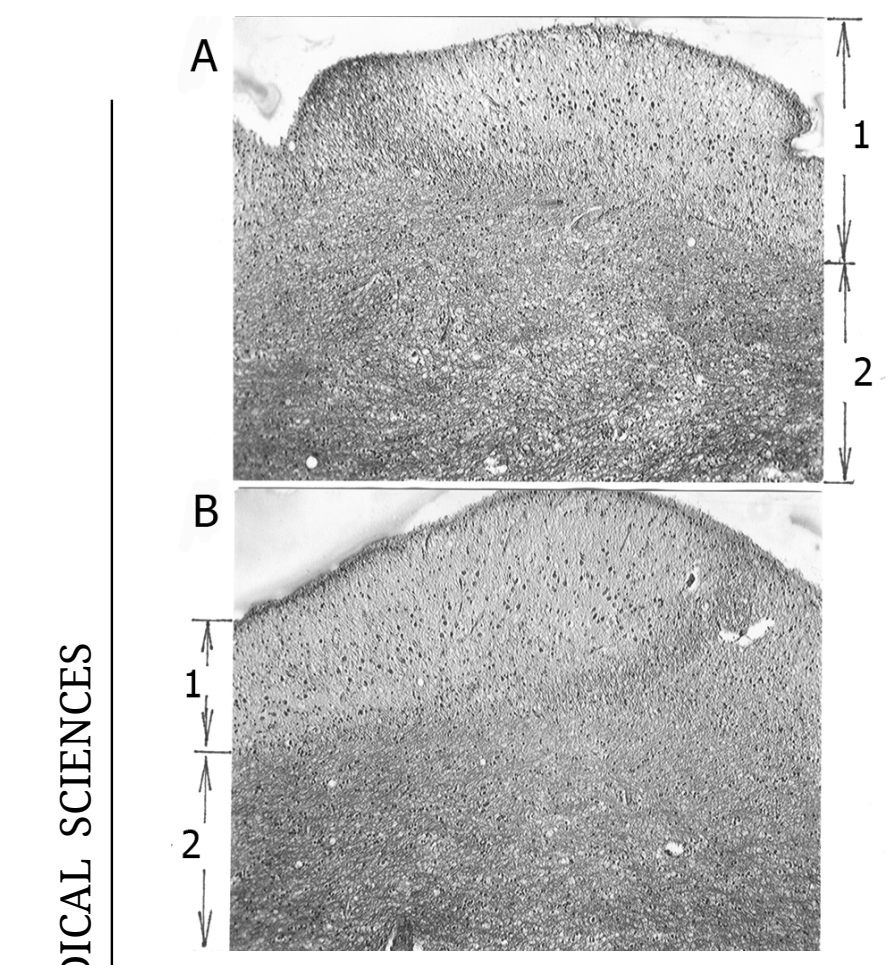

Fig. 3. The medial longitudinal strips of corpus callosum of an adult man (A) and a woman (B) in cross section. Paraffin slices. Van Gieson staining. x10.

1 - medial strips; 2 - the corpus callosum. row band of condensed cellular elements. Inside them there are interfascicular oligodendrocytes appearing not only in the actual corpus callosum, but also in the substance of medial strips, with the only difference: in the latter they are present in more rarefied state. But the most significant feature is that these glial cells are arranged in rows oriented into the thickness of the corpus callosum, thereby creating the idea that both these cells and nerve fibres accompanied by them pass from medial strips to the latter.

\section{Discussion}

This fixed morphological pattern is not enough for unambiguous judgement about the real commutation direction of nerve conductors. So we have to limit ourselves to the general conclusion that medial strips are in close connection with the corpus callosum, either by means of nerve fibres exchange between them, or as a result of their transition from medial longitudinal strips to the corpus callosum. But in fact, in either case it is impossible to establish the proportion of the conductors is involved.

Now let's discuss the lateral longitudinal strips, which are closely adjusted to subjacent matter of the corpus callosum as well as the medial ones, but between them, unlike the former, there is a more pronounced layer of a less densely coloured substance. Paraffin slices, when stained with haematoxylin and eosin by Van Gieson, allow us to define that this enlightened layer entirely relates to the limits of lateral longitudinal strips, because between them and the substance of the corpus callosum there is a dividing strip of compacted substance due to the increased concentration of myelinated nerve fibres in this place (Fig. 4). On the lower microphotograph of this figure one can 

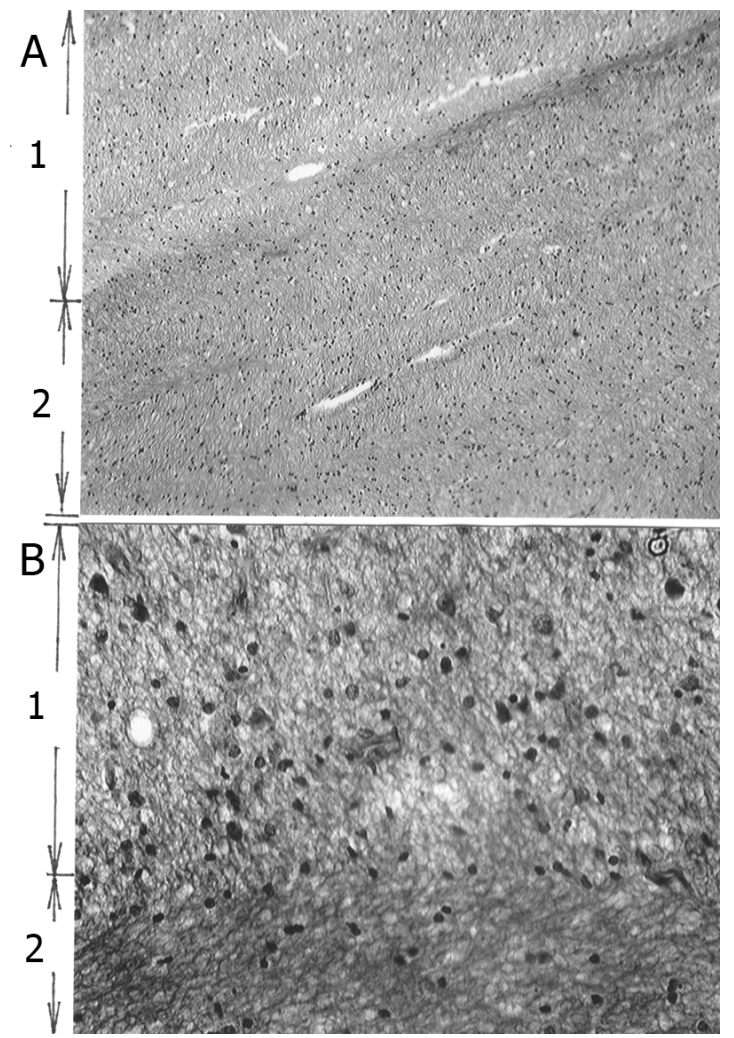

Fig. 4. The border zones between lateral longitudinal strips and the corpus callosum in cross-section. Paraffin slices. A - Van Gieson staining, x10; B -hematoxylin and eosin staining, $x 40$.

1 - lateral strip substance; 2 - the substance of the corpus callosum.

see that this border concentration of nerve fibres seems to appear due to the replenishment of fascicular portions of corpus callosum with nerve conductors penetrating inside them from the enlightened area of lateral strips. In turn, the latter are the nerve fibres, which are separated along their way from the dense mass of longitudinal lateral strips. Although this fact seems obvious, as in the case of medial longitudinal strips, we still have no reason to exclude the fact of their mutual exchange of nerve conductors. Still, on the basis of the data received it is possible to make a presumptive conclusion that lateral longitudinal strips, unlike other similar to them the medial ones, contain a much greater mass of nerve conductors. Most of them in compact form pass in transit manner along limbic ring, while the other part of them, which is less numerous, combines with the mass of nerve fibber's of the corpus callosum commissural cords.
Additionally to this data, it should be noted that both the medial and lateral longitudinal strips are coated with organically connected outer limiting glial membrane, known in the literature as grey coating which has a darker tone compared to white brain matter due to minimum amount of lipoid substances therein in the form of myelin.

\section{Conclusions}

We did not aim at understanding the details of the links between the associative conductors of limbic brain, distributed on lateral and medial longitudinal strips, and the commissural commutations of the corpus callosum, because a research like this requires a lot of efforts, not only by neuromorphologists but also neuroscientists and psychiatrists. The aim of our study was to show that the existing concept of the corpus callosum as a purely adhesive formation between the contralateral cortical areas of the new pallium is far from being complete. The results of our study give evidence that by means of the corpus callosum the connections between conscious and subconscious brain areas are structurally fixed. It can be assumed that the longitudinal strips spanning the corpus callosum on its upper surface are mediatingly related to hippocampal area, related to the formation of ancient pallium. This does not exclude that nerve fibres found in longitudinal strips may have projections on cortical cells of vaulted gyrus, which is considered to be the paleopallium sphere. If that is the case, then the interaction between ancient and old cortex should presumably be carried out by means of lateral longitudinal strips.

But, as mentioned in the introduction, this is not limited to the anatomical structures of limbic brain, having a close relationship with the corpus callosum. Apart from the former (longitudinal strips) and opposite to them, that is, at the bottom side, two other formations are coalesced with the corpus callosum. They are a transparent partition and fornix, which, except for their anatomical descriptions and considering them as conductive constituent of limbic brain, are represented in the literature neither for the presence nor absence of a direct connection of their conductors with commissural fibres of the corpus callosum. Resolving of this issue will be presented in the next report. 


\section{References}

1. Blanchet B, Roland J, Braun M. The anatomy and the MRI anatomy of the interhemispheric cerebral commissures. J Neuroradiol. 1995;22(4): 237-251.

2. Boiagina OD. Sexual dimorphism of the external structure of the trunk of the corpus callosum of middle-aged people. Clinical anatomy and operative surgery. 2016;3(57):27-30.

3. Kostilenko YuP, Bojko IV, Starchenko II. Method of making histological preparations, equivalent thin sections of the large viewing surface, for multi-purpose morphological studies. Morphology. 2007;5:94-96.

4. Luders E, Thompson PM, Toga AW. The development of the corpus callosum in the healthy human brain. J Neurosci. 2010;30(33): 10985-10990.

5. Prakash KN, Nowinski WL. Morphologic relationship among the corpus callosum, fornix, anterior commissure, and posterior commissure MRI-based variability study. Acad Radiol. 2006; 13(1):24-35.

6. Raybaud C. The corpus callosum, the other great forebrain commissures, and the septum pellucidum: anatomy, development, and malformation. Neuroradiology. 2010;52(6):447-477.

7. Roy E, Hague C, Forster B. The corpus callosum: imaging the middle of the road. Can Assoc Radiol J. 2014;65(2):141-147. 